\title{
Spatio-Temporal Scale-Spaces
}

\author{
Daniel Fagerström \\ Computational Vision and Active Perception Laboratory (CVAP) \\ Department of Numerical Analysis and Computing Science \\ KTH (Royal Institute of Technology) SE-100 44 Stockholm, Sweden \\ danielf@nada.kth.se
}

\begin{abstract}
A family of spatio-temporal scale-spaces suitable for a moving observer is developed. The scale-spaces are required to be time causal for being usable for real time measurements, and to be "velocity adapted", i.e. to have Galilean covariance to avoid favoring any particular motion. Furthermore standard scale-space axioms: linearity, positivity, continuity, translation invariance, scaling covariance in space and time, rotational invariance in space and recursivity are used. An infinitesimal criterion for scale-spaces is developed, which simplifies calculations and makes it possible to define scale spaces on bounded regions. We show that there are no temporally causal Galilean scale-spaces that are semigroups acting on space and time, but that there are such scale-spaces that are semigroups acting on space and memory (where the memory is the scale-space). The temporally causal scale-space is a time-recursive process using current input and the scale-space as state, i.e. there is no need for storing earlier input. The diffusion equation acting on the memory with the input signal as boundary condition, is a member of this family of scale spaces and is special in the sense that its generator is local.
\end{abstract}

\section{Introduction}

While "spatial" scale-space theory has become the more or less canonical theory for low-level vision and its concepts, methods and algorithms now are part of the common toolbox in computer vision, there is this far no theory that can serve as a basis for low-level vision for moving images.

The spatio-temporal scale-space theory from Koenderink [11] is based on spatio-temporal convolution on the past image sequence, which makes it computationally heavy and less attractive for practical applications. From a conceptual point of view it is questionable to base a theory on spatio-temporal measurement on the past signal, a real-time system can only have access to the past measurements. The corresponding theory from Lindeberg [12] is more usable in an application setting due to its recursive formulation. On the other hand it is discrete in space-time, which makes it cumbersome to use analytical methods like differential geometry, methods which has been used for deriving a large part of the results in spatial scale-space theory. The spatio-temporal scale-space theory that we propose is both continuous and has a recursive formulation, and thus solves the problems described above. 
In the rest of the article we will start by defining a spatio-temporal scale-space suitable for image motion measurements in the seemingly most natural way: It should be a semigroup on space-time, time causal, covariant with respect to spatial and temporal scale and Galilean boost [7]. We require Galilean covariance as for a moving observer no particular motion or lack of motion should be treated in a special way. It is relative motion that matters. This approach however doesn't work as we show that among the possible Galilean covariant, semigroups on space-time, there are no time causal ones. To simplify proofs and anticipating later needs we also develop infinitesimal criterion on scale-spaces.

After the failure of the "obvious" approach to time causal spatio-temporal scale-space, we perform a deeper analysis of the nature of temporal measurement. And observe that in addition to that a real time system cannot be dependent on future information it cannot be directly dependent on past input either, only its representation of past input, its memory [4]. Based on this insight, we instead define a temporally causal spatio-temporal scale space as a semigroups in space and memory, and derive a family of scale-spaces fulfilling this. If one requires the generator of the scale-space to be local, there is a unique scale-space that is generated by the heat equation.

\section{Generalities}

Our aim with defining a spatio-temporal scale-space is to develop a generic theory about image motion measurements. A measurement is done over an extended point (volume), it is covariant with the relevant symmetry group. It should have the cascade property, i.e. a measurement of a measurement should also be contained in the family of measurements [8]. As the structure of the Galilean similarity group is more complicated than the similarity group that is used in ordinary scale-space theory, some extra care is needed to handle the relation between covariance and the cascade property.

An image is defined as an integrable functions $u \in L_{1}(\Omega)=\Sigma$ over some connected subset of an Euclidean base manifold $\Omega \subset M, M=\mathbb{R}^{d} \ni x=$ $\left(x_{1}, \ldots, x_{d}\right)$ for spatial images and $M=\mathbb{R}^{d+1} \ni x=\left(x_{0}, x_{1}, \ldots, x_{d}\right)$ for spatiotemporal images, where $x_{0}$ is a coordinate in the temporal direction.

Definition 1. For any $u, v \in \Sigma\left(=L_{1}(\Omega)\right)$ and $\alpha, \beta \in \mathbb{R}, \Phi: \Sigma \rightarrow C^{\infty}(\Omega)$ is a point measurement operator if it fulfills:

linearity $\Phi(\alpha u+\beta v)=\alpha \Phi(u)+\beta \Phi(v)$

gray level invariance $\|\Phi u\|_{L_{1}}=\|u\|_{L_{1}}$ positivity $u \geq 0 \Rightarrow \Phi u \geq 0$

point There is a sequence of operators $\mathbb{R}_{+} \ni s \mapsto \Phi_{s}$ s.t. $\lim _{s \rightarrow 0} \Phi_{s} u=u$

Image measurement should respect basic symmetries in the world that is measured. This is described in terms of Lie groups acting on images. Let $g \in G$, where $G$ is a Lie group, then the group act on the base space as $g \mapsto g \cdot x=T_{g} x$, where $T_{g}: \operatorname{Diff}(\Omega)$, (i.e a diffeomorphism on $\Omega$ ) and on images and measurements like $T_{g} u(x)=u(g \cdot x)$. 
Ideally we would like point measurement operators that are invariant, $\Phi T_{g}=$ $\Phi$, with respect to the group but for many groups (e.g. the scaling group) that is not possible. We have to be content with requiring covariance, i.e. we have a family $H \ni h \mapsto \Phi_{h}$ of measurement operators, fulfilling,

$$
T_{g} \Phi_{h}=\Phi_{g \cdot h} T_{g}
$$

Definition 2. We call such a family of point measurement operators a G-covariant point measurement space.

From the definition it can easily be shown that the action $G \times H \ni(g, h) \mapsto$ $g \cdot h=\sigma(g, h)=\sigma_{g}(h) \in H$ is a Lie group action on the set $H$. As a smaller set of measurement operators is easier to manage we prefer measurement spaces that are invariant to as large subgroup as possible.

For the spatio-temporal scale-space we will require covariance with respect to the $(n+1)$-dimmensional Galilean similarity group that in matrix form becomes:

$$
\left(\begin{array}{c}
t^{\prime} \\
x^{\prime}
\end{array}\right)=\left(\begin{array}{cc}
\tau & 0 \\
v & \sigma R
\end{array}\right)\left(\begin{array}{l}
t \\
x
\end{array}\right)+a
$$

where $x, v \in \mathbb{R}^{n}, t \in \mathbb{R}, R \in S O(n), \sigma, \tau \in \mathbb{R}_{+}$and $a \in \mathbb{R}^{n+1}$.

Now we have a family of measurement devices $\Phi_{h}$ that is covariant under the chosen Lie group. But we still don't know how the actual measurements, $\Phi_{h} u$ are related. Considering that the result of a measurement can be measured in turn it is natural to require the family of measurements to be closed under composition,

$$
\Phi_{h_{1}} \Phi_{h_{2}} u=\Phi_{h_{1} \cdot h_{2}} u,
$$

where $H \times H \ni\left(h_{1}, h_{2}\right) \mapsto h_{1} \cdot h_{2} \in H$, is an abstract Lie semigroup. Although there is a rich modern theory about Lie semigroups, it will for our needs be enough to consider Lie semigroups $H \subset G$ that are subsets of a Lie group $(G, \cdot)$ and closed under composition but not necessarily under inversion. A simple example is $\left(\mathbb{R}_{+},+\right)$.

From (3) it can be seen that $\left\{\Phi_{h} \mid h \in H\right\}$ is an operator semigroup and furthermore as $H \ni h \mapsto \Phi_{h}$ is a Lie semigroup homomorphism, the operator semigroup must be a Lie semigroup as well. Combining $G$-covariance with the cascade property we finally get:

Definition 3. A G scale-space is a minimal semigroup of $G$-covariant point measurements, i.e. a family of operators $H \ni h \mapsto \Phi_{h}: \mathrm{L}\left(\Sigma, C^{\infty}(\Omega)\right)$ fulfilling

$$
T_{g} \Phi_{h} T_{g^{-1}}=\Phi_{g \cdot h},
$$

a slight reorganization of (1) to emphasize how $G$ act on the semi group of operators and (3), and where $G$ is an abstract Lie group and $H$ an abstract Lie semigroup.

It can be seen that $G \times H \ni(g, h) \mapsto g \cdot h=\sigma(g, h) \in H$ is both a group homomorphism in the first argument and a semigroup homomorphism in the second. When the homomorphism is trivial in the first argument we have an invariant operator, $T_{g} \Phi_{h} T_{g^{-1}}=\Phi_{h}$. 


\section{Infinitesimal generators}

In preparation for later needs we derive infintesimal conditions for a $G$ scalespace. There are several advantages in doing that. It is more realistic in the sense that we mainly care for local symmetries. It allows for more general boundary conditions, e.g. for a bounded sensor and it simplifies calculations as it linearizes the problem.

The infinitesimal object that corresponds to a Lie group $G$ is a Lie algebra, $\mathcal{L} G=\mathfrak{g}$, which is the linear space of infinitesimal generators of the group at identity together with an anti-commutative bilinear operator $\mathfrak{g} \times \mathfrak{g} \ni(v, w) \mapsto$ $[v, w] \in \mathfrak{g}$, the Lie bracket (see e.g. [14] for details). The transformation group $g \mapsto T_{g}$ corresponds to the Lie algebra of infinitesimal generators $\mathfrak{g} \ni v \mapsto A_{v}=$ $A(v)$ where $A=d T(e): \mathfrak{g} \rightarrow \mathrm{L}(\Sigma, \Sigma)$. Choosing a base $\left\{v_{1}, \ldots, v_{n}\right\} \subset \mathfrak{g}$ for $\mathfrak{g}$ the corresponding base for the infinitesimal generators of the transformation group is $\left\{A_{1}, \ldots, A_{n}\right\}$ where $A_{j}=A_{v_{j}}$

For a Lie semigroup $H$ the infinitesimal object is a Lie wedge, $\mathcal{L} H=\mathfrak{h}$, and is a closed cone in a Lie algebra. The properties for Lie algebras mentioned above also holds for Lie wedges with the restriction that it is not necessary for both $[v, w]$ and $[w, v]$ for $v, w \in \mathfrak{h}$ to be members of the wedge [9]. Only Lie semigroups that are generated by their Lie wedge will be considered, i.e. $\exp \left(\mathbb{R}_{+} \mathfrak{h}\right)=H$.

From a $G$ scale-space, using $\Phi(h)(x)=\Phi_{h}(x)$, for emphasizing the dependency on the semigroup $H$, the corresponding infinitesimal operator is $B=$ $d \Phi(e): \mathfrak{h} \rightarrow \mathrm{L}(\Sigma, \Sigma), \mathfrak{h} \ni w \mapsto B_{w}=B(w)$. Choosing a base $\left\{w_{1}, \ldots w_{m}\right\} \subset \mathfrak{h}$

for the Lie wedge a corresponding base, $\left\{B_{1}, \ldots, B_{m}\right\}$ where $B_{k}=B_{w_{k}}$, of the Lie wedge of infinitesimal generators for the semi group is given.

\subsection{Covariant generators}

The infinitesinal form of the covariance equation (4) is,

$$
\left[A_{v}, B_{w}\right]=B_{C(v, w)},
$$

where $\mathfrak{g} \times \mathfrak{h} \ni(v, w) \mapsto C(v, w) \in \mathfrak{h}$ is the differential of $\sigma$ (from (4)) with respect to both arguments. This can be shown by calculating the differential to the equation both with respect to the group $G$ and the semigroup $H$. An operator $B_{w}$ fullfilling such an equation is called a covariant tensor operator [1] in mathematical physics. The operator $C$ is denoted the covariance tensor and is a Lie algebra representation in the first argument and a Lie wedge representation in the second.

In coordinate form the infinitesimal covariance equation becomes:

$$
\left[A_{j}, B_{k}\right]=\sum_{l} C_{j, k l} B_{l}
$$

where $C\left(v_{j}, w_{k}\right)=C_{j}\left(w_{k}\right)=\sum_{l} C_{j, k l} w_{l}$. For an infinitesimally invariant operator, $\left[A_{v}, B_{w}\right]=0$. Note that requiring an operator $B_{w}$ to be infinitesimally 
invariant with respect to an action $A_{v}$ means for the covariance tensor that $C(v, w)=0$.

Now we take a look at the semigroup generated by a set of covariant tensor operators. A one-parameter Lie semigroup is generated by an element in the corresponding Lie wedge $w \in \mathfrak{h}$ by $\mathbb{R}_{+} \ni s \mapsto \exp (s w)=h_{w}(s) \in H_{w} \subset H$. Let $u: H \times \Omega \rightarrow \mathbb{R}$ and set $\mathfrak{h} \ni w \mapsto u_{w}(s, x)=u\left(h_{w}(s), x\right)$,

$$
\left\{\begin{array}{l}
\partial_{s} u_{w}=B_{w} u_{w} \\
u_{w}(e, x)=f(x),
\end{array}\right.
$$

where $f \in \Sigma$. For an abstract Cauchy problem, like (7), the solution can be described in terms of a semigroup $u_{w}(s, x)=\exp \left(s B_{w}\right) f(x)=\Phi_{h_{w}(s)} f(x)$. And given that we have chosen semigroups $h \in H$, such that $h=\exp (s w)$ for some $s \in \mathbb{R}_{+}, w \in \mathfrak{h}$, we have [9],

$$
u(h, x)=\Phi_{h} f(x) .
$$

Reconnecting to the $G$ scale-space axioms, Definition 3, the Cauchy problem (7) for a $\mathfrak{g}$ tensor operator generates a $G$-covariant semigroup. Furthermore, looking at Definition 1, the semigroup will be linear as long as the infinitesimal generator $B_{w}$ is independent of the evolution parameter $(s$ in $(7))$. Also the point property follows from the boundary condition in $(7)$. What is left to give an infinitesimal characterization of is the positivity and gray level invariance properties.

\subsection{Pseudo differential operators}

For being able to continue we must be more concrete about the form of the operators in (5). The infinitesimal generators of the transformation group are on the form: $\sum_{j \leq n} a_{j}(x) \partial_{j}$, where $a_{j}: M \rightarrow \mathbb{R}$. In earlier work in scale-space theory the infinitesimal generator have been the inverse Fourier transform of some smooth function. By using pseudodifferential operators, $\Psi \mathrm{DO},[3]$ we get a large enough class of operators to embed both of them. $\Psi$ DO's are defined by

$$
A u(x)=(2 \pi)^{-n} \int e^{i x \cdot \xi} a(x, \xi) \tilde{u}(\xi) d \xi,
$$

where $\tilde{u}(\xi)=\int e^{-i x \cdot \xi} u(x) d x$ and $a(x, \xi)=\sum_{|\alpha| \leq m} a_{\alpha}(x) \xi^{\alpha}$, and is called the symbol of $A$. The corresponding operator is denoted $a(x, D)$.

The composition of two symbols is:

$$
c(x, \xi)=a(x, \xi) \circ b(x, \xi)=\sum_{\alpha} \frac{1}{\alpha !}\left[\partial_{\xi}^{\alpha} a(x, \xi)\right] \cdot\left[D_{x}^{\alpha} b(x, \xi)\right],
$$

where $D_{j}=i^{-1} \partial_{j}$ and the commutator determines a Lie algebra structure on the symbols.

In this paper we will only consider translation invariant scale-spaces which further restricts the form of the symbol. A base for the Lie algebra of translations is, $\mathfrak{t}(n)=\left\{\partial_{1}, \ldots, \partial_{n}\right\}$, and the corresponding symbols are, $\partial_{j} \sim i^{-1} \xi_{j}$. 
Lemma 1. Translation invariant symbols are position independent, $b(x, \xi)=$ $b(\xi)$.

Proof. An infinitesimally translational invariant symbol $b$ fulfills, $\left[i^{-1} \xi_{j}, b(x, \xi)\right]=$ 0 , for $j \leq n$. Leading to, $\partial_{x_{j}} b(x, \xi)=0$, for all $j \leq n$, i.e. the symbol is independent of $x$.

We can also see that any two translationally invariant symbols $b_{i}(\xi), b_{j}(\xi)$ commutes, $\left[b_{i}(\xi), b_{j}(\xi)\right]=0$, which means that cones of translation invariant symbols automatically becomes Lie wedges.

\subsection{Positivity and gray level invariance}

A positive translation invariant linear operator has a positive kernel. It can be shown that a kernel $\Omega \ni x \mapsto \phi(x)$, is positive iff its Fourier transform is positive definite, i.e. for each $k \in \mathbb{N}$ and each set of $\xi_{1}, \ldots, \xi_{k} \in \mathbb{R}^{d}$ the matrix $\left(\tilde{\phi}\left(\xi_{i}-\xi_{j}\right)\right)_{i, j=1, \ldots, k}$ is positive Hermitian, (e.g. [10]). Furthermore for kernels that generate a semigroup $\mathbb{R}_{+} \ni s \mapsto \phi_{s}$, their Fourier transform is $\tilde{\phi}_{s}(\xi)=e^{-s b(\xi)}$, where $\mathbb{R}^{d} \ni \xi \mapsto b(\xi) \in \mathbb{C}$.

Definition 4. A symbol $\mathbb{R}^{d} \ni \xi \mapsto b(\xi) \in \mathbb{C}$ is negative definite if the matrix $\left(b\left(\xi_{i}\right)+\overline{b\left(\xi_{j}\right)}-b\left(\xi_{i}-\xi_{j}\right)\right)_{i, j=1, \ldots, k}$ is positive Hermitian for any choice of $k \in \mathbb{N}$ and each set of $\xi_{1}, \ldots, \xi_{k} \in \mathbb{R}^{d}$.

A kernel is positive definite iff its symbol is negative definite [10]. For negative definite symbols $b(\xi), b(0) \geq 0$ and they have at most quadratic growth at $\infty$ i.e. $|b(\xi)| \leq k_{b}\left(1+|\xi|^{2}\right)$ for some $k_{b} \in \mathbb{R}_{+}$.

For gray level invariance we use an adaption of a theorem from [10].

Theorem 1. For a gray level invariant semigroup $\Phi_{s}$ generated by the $B$ with symbol $b$ the following holds: $\Phi_{s} 1=1$ for all $s \geq 0, B 1=0$ and $b(0)=0$ where $\Omega \ni x \mapsto 1(x)=1$.

A generator such that $B 1=0$ is said to be conservative.

\subsection{Infinitesimal $G$ scale-space}

Combining our results about infinitesimal generators of a $G$ scale-space, we can now state:

Definition 5. A $\mathfrak{g}$ scale-space wedge, is a minimal Lie wedge of negative definite conservative operators $\mathfrak{h} \ni w \mapsto B_{w}: \mathrm{L}(\Sigma, \Sigma)$, that is a covariant tensor operator (5), with respect on the Lie algebra action $\mathfrak{g} \ni v \mapsto A_{v}: \mathrm{L}(\Sigma, \Sigma)$ and the Lie algebra and Lie wedge representation $(v, w) \mapsto C(v, w)$.

Summarizing the discussion above:

Theorem 2. A G scale-space is generated (using (7)) by its corresponding $\mathfrak{g}$ scale-space wedge. 


\section{Scale-space generators}

Now we have the tools we need for being able to study infinitesimal G-scale spaces. We will apply these tools for the affine line, Euclidean similarity spaces, and Galilean spaces with scaling in time and space.

\subsection{The affine line}

A one dimensional scale space measurement is invariant with respect to translation and covariant with respect to scaling. The affine line has the infinitesimal generators,

$$
\mathfrak{g l}(1)=\mathfrak{t}(1) \cup\left\{x \partial_{x}\right\},
$$

commutator $\left[\partial_{x}, x \partial_{x}\right]=\partial_{x}$, and the symbol for scaling is, $i^{-1} x \xi$.

Lemma 2. Translation invariant symbols $b: \mathbb{R} \rightarrow \mathbb{C}$ covariant with $\mathfrak{g l}(1)$ are on the form, $b(\xi)=k \xi^{\alpha}$ for any $k \in \mathbb{C}$ and $\alpha \in \mathbb{R}$.

Proof. From translation invariance, the covariance tensor must obviously be trivial for the translation generator, for scaling the simplest non-trivial representation is $C\left(x \partial_{x}\right)=\alpha$, for any $\alpha \in \mathbb{R}$. Combining this with, (5) we have, $\left[i^{-1} x \xi, b(\xi)\right]=\alpha b(\xi)$, for the symbol $b(\xi)$. Using (10), we get $\xi b^{\prime}(\xi)=\alpha b(\xi)$ which has solutions on the form given in the lemma.

Parameterizing $k$ as $k=c e^{i \theta \pi / 2}$, where $c, \theta \in \mathbb{R}$ and disregarding the, in this context, uninteresting scaling parameter $c$, we have:

Definition 6. The Feller fractional derivative [17] is defined as,

$$
D_{\theta}^{\alpha}(\xi)=-e^{i \theta \pi / 2} \xi^{\alpha}=-|\xi|^{\alpha} e^{i \operatorname{sign}(\xi) \theta \pi / 2},
$$

where $0<\alpha \leq 2, \alpha \neq 1$ and $|\theta| \leq \alpha$ for $0<\alpha<1$ and $|\theta| \leq 2-\alpha$ for $1<\alpha \leq 2$.

The symmetric part $D_{0}^{\alpha}=D^{\alpha} \sim|\xi|^{\alpha}$ is called the Riesz fractional derivative. For $\xi \in \mathbb{R}^{d}$ the notation $-(-\Delta)^{\alpha / 2}=D^{\alpha}$, is also used for emphasizing that the derivative can be seen as a generalization of the Laplacian.

Decomposed as a linear combination of the two one sided operators, $D_{\theta}^{\alpha}(\xi)=$ $-c_{+}(\alpha, \theta) D_{+}^{\alpha}(\xi)-c_{-}(\alpha, \theta) D_{-}^{\alpha}(\xi)$, the operator $D_{+}^{\alpha}\left(D_{-}^{\alpha}\right)$ is called the left (right) sided Riemann Liouville fractional derivative and is given by,

$$
D_{ \pm}^{\alpha}(\xi)=(\mp i \xi)^{\alpha}=|\xi|^{\alpha} e^{\mp i \operatorname{sign}(\xi) \alpha \pi / 2}, \quad c_{ \pm}(\alpha, \theta)=\frac{\sin [(\alpha \mp \theta) \pi / 2]}{\sin (\alpha \pi)}
$$

It can be shown that the Feller derivative is negative definite and conservative for the values of $\alpha, \theta$ given in the definition. And from this we have:

Theorem 3. A gll(1) scale-space wedge is generated by a Feller fractional derivative $D_{\theta}^{\alpha}$ with parameters according to the definition. The subspace of reflection symmetric wedges are generated by Riesz fractional derivatives $D^{\alpha}$ with $0<\alpha \leq 2$ and temporally causal wedges by left sided Riemann Liouville fractional derivatives $D_{+}^{\alpha}$ with $0<\alpha<1$. 
The three parameter family of functions generated of the Feller derivative are called stable densities [5] and appear in generalizations of the central limit theorem. The stable density for $\alpha=2, \theta=0$ is the normal distribution, for $\alpha=1, \theta=0$ it is the Cauchy distribution and for $\alpha=\theta=1 / 2$ is the solution of the signaling equation, i.e. diffusion on the half line with the signal as input at the end. Symmetric stable densities, $\theta=0$ was the result of the scale space axiomatization in $[15,2]$. The maximally asymmetric, extremal stable density functions are one sided for $0<\alpha<1$ and $\theta= \pm \alpha$, these where the result of an axiomatization of scale spaces with temporal causality in [4].

In $[15,2]$ these result are extended to the Euclidean similarity group, which on $\mathbb{R}^{2}$ consists of translation in the plane, rotation and scaling.

Theorem 4. A Euclidean similarity $\mathfrak{e s}(2)=\mathfrak{t}(2) \cup \mathfrak{s}(2) \cup \mathfrak{s o}(2)$ scale-space wedge is generated for any $0<\alpha \leq 2$ by the Riesz fractional derivative $-(-\Delta)^{\alpha / 2}$.

Where $\mathfrak{s}(2)=\left\{x_{1} \partial_{1}+x_{2} \partial_{2}\right\}$ is the scaling generator is and $\mathfrak{s o}(2)=\left\{x_{2} \partial_{1}-x_{1} \partial_{2}\right\}$ is the rotation generator.

\subsection{Galilean Similarity}

The $1+1$ dimensional Galilean similarity group, i.e. translation invariance in space and time, separate scaling in space and time and Galilean boost in space time, have the following set of infinitesimal generators,

$$
\gamma \mathfrak{s}(2)=\mathfrak{t}(2) \cup \mathfrak{s}(1) \oplus \mathfrak{s}(1) \cup \gamma(1),
$$

where $\gamma(1)=\left\{\gamma=x_{0} \partial_{1}\right\}$ is the Galilean boost that "skew" space-time and $\mathfrak{s}(1) \oplus \mathfrak{s}(1)$ is a direct sum of the scaling generator in space and time respectively. The non-zero commutators are $\left[\partial_{j}, x_{j} \partial_{j}\right]=\partial_{j},\left[\partial_{0}, \gamma\right]=\partial_{1},\left[x_{0} \partial_{0}, \gamma\right]=\gamma$ and $\left[x_{1} \partial_{1}, \gamma\right]=-\gamma$.

Theorem 5. A $\gamma \mathfrak{s}(2)$ scale-space wedge is generated by $\left\{\partial_{0}^{2}, \partial_{0} \partial_{1}, \partial_{1}^{2}\right\}$.

Proof. Requiring our Lie wedge separately covariant with respect to scaling booth in space and time and using the results from scale space on the affine line we can see that any wedge must contain two generators on the form $b_{j}\left(\xi_{0}, \xi_{1}\right)=$ $k_{j} \xi_{j}^{\alpha_{j}}, j=0,1$. Applying the Galilean boost, which has the symbol $\gamma=i^{-1} x_{0} \xi_{1}$ on these the spatial generator disappears $\left[\gamma, k_{1} \xi_{1}^{\alpha_{1}}\right]=0$, while repeated application of the Galilean boost on the temporal generator gives, $\operatorname{ad}\left(i^{-1} x_{0} \xi_{1}\right)^{l}\left(k_{0} \xi_{0}^{\alpha_{0}}\right)=$ $k_{0}\left(\prod_{j=0}^{l-1} \alpha_{0}-j\right) \xi_{0}^{\alpha_{0}-l} \xi_{1}^{l}$, where $\operatorname{ad}(a)(b)=[a, b]$. To get a finite base of generators we must have $\alpha_{0} \in \mathbb{Z}_{+}$, furthermore to generate a positive semigroup $\alpha_{0}, \alpha_{1} \leq 2$. For $\alpha_{0}=2$ we get the set of symbols, $\left\{\xi_{0}^{2}, \xi_{0} \xi_{1}, \xi_{1}^{2}\right\}, k_{0} \in \mathbb{R}$ for $\alpha=2$ (then only $\theta=0$ is allowed). This set of generators are booth closed under the Galilean similarity group and complete by choosing $\alpha_{1}=2, k_{1}=1$ for the spatial generator.

It should be noted that the generated scale-space is symmetric booth in time and space and thus no time causal scale-spaces are given with this axiomatization. And as $\gamma \mathfrak{s}(n), n \geq 2$ have $\gamma \mathfrak{s}(2)$ as a sub algebra, no time causal scale-spaces are possible for them either. 


\section{Time causal Galilean scale-spaces}

This far we have seen that Galilean scale-spaces as we have defined them have kernels that are symmetric in the temporal direction. This means that both the past and the future signal is used for the measurement, this is a disappointment if we want to use such a scale space for real time measurements.

Definition 7. The history at time $t$ of the spatio-temporal signal $\mathbb{R} \times \mathbb{R}^{d} \ni$ $(t, x) \mapsto f(t, x) \in \mathbb{R}$ is, $f(t, s, x)=f(t-s, x)$ when $s<t$ and 0 otherwise. $A$ time causal measurement operator only depends on the history at time $t$ for a measurement at $t$.

For a real time system temporal causality is obviously necessary. But as discussed in [4] defining temporal measurement in terms of a convolution or a evaluation equation on the history is to beg the question. It would require the measurement device to already have access to what it is supposed to measure.

This conceptual problem can be resolved by involving a memory of the history in the definition of temporal measurement. The measurement device should only have access to the current signal and its memory of previous measurements. As the memory is supposed to represent the history it is reasonable to make it as similar to the history as possible. This can be done by requiring the memory to be a half-space of the same dimensions as the history and subject to the same symmetry requirements.

We apply these considerations on the pure time causal scale-space (with no spatial dimensions).

Definition 8. A time causal scale-space on the affine line $\mathbb{R} \times \mathbb{R}_{+} \ni(t, \tau) \mapsto$ $u(t, \tau)$, where $t$ is the temporal coordinate and $\tau$ the memory coordinate, is generated by the signaling problem,

$$
\left\{\begin{array}{l}
\partial_{t} u=B u \\
u(t, 0)=f(t)
\end{array}\right.
$$

where the operator $B$ is independent of time and $f: \Sigma$ is the input signal. The measurement operator implicitly defined by $u(t, \tau)=\Phi_{\tau} f(t)$ is a $G L(1)$ covariant point measurement operator (but not necessarily a semigroup) and the infinitesimal generator $B$ is a $\mathfrak{g l}(1)$ scale-space wedge.

Theorem 6. A time causal scale-space on the affine line is generated by the right sided Riemann Liouville fractional derivative $D_{\tau-}^{\alpha}$, with $1<\alpha \leq 2$ (where the suffix $\tau$ denotes that it is applied on the memory domain).

Proof. The form of the generators of the $\mathfrak{g l}(1)$ scale-space wedge is given in Theorem 3. Of the generators given there, only $B=D_{\tau-}^{\alpha}, 0<\alpha \leq 2$ are translation invariant on the right (positive) half line, as all other generators have support on the left half line as well (for non integer differentiation order).

In [4] it is shown that (15), with $B=D_{\tau-}^{\alpha}$ is equivalent to $\partial_{\tau} u=-D_{t+}^{1 / \alpha} u$ with initial value $u(t, 0)=f(t)$, where $0<1 / \alpha<1$ that is $\alpha>1$ for $\Phi_{\tau}$ to be a time causal scale space. 
It is worth noticing that the only local generator for this family of scale spaces is $D_{\tau-}^{2}=\partial_{\tau}^{2}$, which means that in this case the scale space is generated by the heat equation, although with different boundary conditions compared to ordinary spatial scale space.

\subsection{Galilean similarity}

Now we extend these results to Galilean space-time. The time causal Galilean scale-space is as discussed above a scale space on space and memory rather than on space and time. Something new compared to the the previously discussed scale-spaces is that for the generator of the scale space on memory $\partial_{t}=D_{\tau-}^{\alpha}$, the symmetry Lie algebra will not only act on the generator but on the time derivative $\partial_{t}$ as well.

Definition 9. Let $\gamma \mathfrak{s}(d+1)=\mathfrak{e s}(d) \oplus \mathfrak{g l}(1) \cup \gamma(d)$. The $d+1$-dimensional time causal Galilean scale-space $\mathbb{R}_{+} \times \mathbb{R}^{d} \times \mathbb{R}_{+} \times \mathbb{R} \times \mathbb{R}^{d} \ni(\sigma, v, \tau, t, x) \mapsto$ $u(\sigma, v, \tau, t, x) \in \mathbb{R}$, where $\sigma$ is spatial scale, $v$ is velocity, $\tau$ is memory (and temporal scale) is a $\gamma \mathfrak{s}(d+1)$-covariant, point measurement space in space-time $(t, x)$ and a $\mathfrak{g l}(1)$-wedge in memory $\tau$.

Theorem 7. A $d+1$-dimensional time causal Galilean scale-space (for $d=1,2$ ) is generated by the evolution equation,

$$
\left\{\begin{array}{l}
\partial_{t} u=-v \cdot \nabla_{x} u+D_{\tau-}^{\alpha_{0}} u \\
\partial_{\sigma} u=-\left(-\Delta_{x}\right)^{\alpha / 2} u \\
u(0,0,0, t, x)=f(t, x)
\end{array}\right.
$$

where $1<\alpha_{0} \leq 2,0<\alpha \leq 2, \sigma$ is the spatial scale direction, $v \in \mathbb{R}^{d}$ the velocity vector, $\partial_{s}=\partial_{t}+v \cdot \nabla_{x}$ is the spatio-temporal direction, $\nabla_{x}=\left(\partial_{1}, \ldots, \partial_{d}\right)$ is the spatial gradient and $\Delta_{x}$ is the spatial Laplacian.

The equation,

$$
\left\{\begin{array}{l}
\partial_{t} u=-v \cdot \nabla_{x} u+\partial_{\tau}^{2} u \\
\partial_{\sigma} u=\Delta_{x} u \\
u(0,0,0, t, x)=f(t, x),
\end{array}\right.
$$

is unique in the family of evolution equations as it is the only one that has local generators.

Proof. First it is shown in Theorem 4 that the spatial part of the scale space is generated by $\partial_{\sigma} u=-\left(-\Delta_{x}\right)^{\alpha / 2} u, 0<\alpha \leq 2$ that besides being covariant with $\mathfrak{e s}(2)$ is invariant with respect to temporal translation and scaling and spatiotemporal Galilean boost. For the temporal part of the scale-space we know from Theorem 6 that the generator $\partial_{t} u=D_{\tau-}^{\alpha_{0}} u, 1<\alpha \leq 2$ is a $\mathfrak{g l}(1)$-wedge. As $D_{\tau-}^{\alpha_{0}}$ is independent of space and time it is obviously invariant with the Galilean similarity group. But $\partial_{t}$ is not checking for the commutation relations with $\gamma \mathfrak{s}(d+1)$ the non zero commutators are for scaling $\left[\partial_{t}, t \partial_{t}\right]=\partial_{t}$ and for the Galilean boosts $\left[\partial_{t}, t \partial_{j}\right]=\partial_{j}, i=1,2$. Checking $\partial_{j}$ for all commutators no further generators are added, so $\left\{\partial_{t}, \partial_{j}\right\}$ is closed under $\gamma \mathfrak{s}(d+1)$. As a result, 
linear combinations of $\left\{\partial_{j}\right\}$, that is $v \cdot \nabla_{x}, v \in \mathbb{R}^{d}$ must be added to the temporal scale-space generator to make it closed under the required symmetries.

While there is no closed form for the general causal Galilean scale-space, it can be shown that (17) have the solution

$$
\begin{aligned}
& u(\sigma, v, \tau, t, x)=\phi(\sigma, v, \tau, \cdot, \cdot) * f(t, x), \\
& \phi(\sigma, v, \tau, t, x)=\frac{\tau \exp \left(-\frac{\tau^{2}}{4 t}-\frac{(x-t v) \cdot(x-t v)}{4 \sigma}\right)}{\sqrt{4 \pi} t^{3 / 2}(4 \pi \sigma)^{d / 2}} .
\end{aligned}
$$
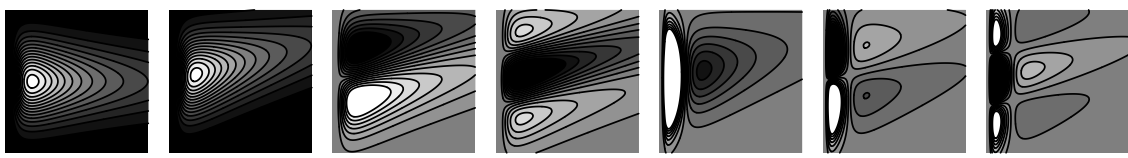

Fig. 1. Causal Galilean scale-space: space vertically and time horizontally, from left to right: two different velocity adaptations of $u$, then $u_{x}, u_{x x}, u_{t}, u_{x t}, u_{x x t}$

\section{Discussion}

The main result of this article is that we have shown (Theorem 7), that there is a reasonable spatio-temporal velocity adapted scale space for an active observer. It is booth time causal and recursively generated, in the sense that it only depends on current input and its memory, not the history of the input. The set of axioms is very close to modern scale-space axiomatizations [18], but with the main difference that we apply them on space and memory instead of space and time.

Comparing to earlier formulations of time causal Galilean scale spaces Lindeberg's [12] is close in the sense that it is a recursive formulation, but the formulation is discrete so covariances are only approximate and it is much harder analyze the properties. The approach from Florack [6] is based on a Gaussian Galilean scale-space that is made time causal by doing a logarithmic transformation of the time domain, it depends on the history of the signal and no recursive formulation has been suggested. Salden [16] have proposed a time causal spatiotemporal scale-space where the diffusion equation is applied (separately on the spatial and temporal domain) on the history of the signal so it is obviously dependent on history rather than recursive. Although the original formulation is not Galilean, it could easily be, by using a Galilean transformation on the generator.

For numerical implementation of the time causal Galilean scale-space the heat equation scheme (17) is most attractive as the fractional derivatives are integral operators and need to involve a much larger number of grid points for getting satisfying precision. For the heat equation it should be noted that it 
consists of two independent heat equations one in space and one in space time. The one in space can be computed with an explicit scheme for the heat equation with sub sampling as described in [13]. For the spatio-temporal part the scheme in [4] can be used. This makes the proposed scale-space highly efficient as only a two or three point derivative kernel needs to be applied in the temporal direction instead of a full temporal convolution kernel.

\section{References}

1. A. O. Barut and R. Raczka. Theory of Group Representaions and Applications. World Scientific, 2 edition, 1986.

2. R. Duits, L. Florack, J. de Graaf, and B. ter Haar Romeny. On the axioms of scale space theory. J. of Mathematical Imaging and Vision, 20(3):267-298, May 2004.

3. Y. Egorov and S. M.A., editors. Partial Differential Equations II, volume 31 of Encyclopaedia of Mathematical Sciences. Springer Verlag, Berlin, 1994.

4. D. Fagerström. Temporal scale spaces. International Journal of Computer Vision, 64(2/3):97-106, 2005.

5. W. Feller. An introduction to probability theory and its application, volume 2. John Willey \& Sons, Inc., 1966.

6. L. Florack. Visual representations embodying spacetime structure. Technical Report UU-CS-1999-07, Utrecht University, 1999.

7. L. Florack, B. ter Haar Romeny, J. Koenderink, and M. Viergever. Scale and the differential structure of images. Image and Vision Computing, 10:376-388, 1992.

8. L. M. J. Florack. Image Structure. Series in Mathematical Imaging and Vision. Kluwer Academic Publishers, Dordrecht, Netherlands, 1997.

9. E. Hille and R. S. Phillips. Functional analysis and semi-groups. American Mathematical Society, 1957.

10. N. Jacob. Pseudo-Differential Operators and Markov Processes. Number 94 in Mathematical Research. Akademie Verlag, Berlin, 1996.

11. J. Koenderink. Scale-time. Biological Cybernetics, 58:169-162, 1988.

12. T. Lindeberg. Time-recursive velocity-adapted spatio-temporal scale-space filters. In P. Johansen, editor, Proc. 7th European Conference on Computer Vision, volume 2350 of Lecture Notes in Computer Science, pages 52-67, Copenhagen, Denmark, May 2002. Springer Verlag, Berlin.

13. T. Lindeberg and L. Bretzner. Real-time scale selection in hybrid multi-scale representations. In L. Griffin, editor, Proc. Scale-Space'03, volume 2695 of Lecture Notes in Computer Science, pages 148-163, Isle of Skye, Scotland, June 2003. Springer-Verlag.

14. A. L. Onishchik, editor. Lie Groups and Lie Algebra I, volume 20 of Encyclopaedia of Mathematical Sciences. Springer-Verlag, 1993.

15. E. Pauwels, L. VanGool, P. Fiddelaers, and T. Moons. An extended class of scaleinvariant and recursive scale space filters. PAMI, 17(7):691-701, July 1995.

16. A. Salden, B. ter Haar Romeny, and M. Viergever. Linear scale-space theory from physical principles. JMIV, 9(2):103-139, September 1998.

17. S. G. Samko, A. A. Kilbas, and O. I. Marichev. Fractional integrals and derivatives : theory and applications. Gordon and Breach Science Publishers, cop., Yverdon, 1992.

18. J. Weickert, S. Ishikawa, and A. Imiya. Linear scale-space has first been proposed in japan. J. of Mathematical Imaging and Vision, 10:237-252, 1999. 